\title{
Acoustics in water: synergies with marine biology
}

\author{
Mike van der Schaar ${ }^{1, \star}$, Robert Lahmann², Kay Graf ${ }^{2}$, Ludwig Houégnigan ${ }^{1}$, Alba Solsona ${ }^{1}$, \\ Steffen De Vreese ${ }^{1}$, Antonio M. Sánchez ${ }^{1}$, and Michel André ${ }^{1}$ \\ ${ }^{1}$ Laboratory of Applied Bioacoustics, UPC, Barcelona, Spain. \\ ${ }^{2}$ Erlangen Centre for Astroparticle Physics (ECAP), Friedrich-Alexander-Universität, Erlangen, Germany.
}

\begin{abstract}
This paper presents some of the bioacoustics related analysis that was performed on the ANTARES data, focussing on the year 2014. The data was processed for sperm whale, dolphin and shipping presence and grouped by hour of the day. It seemed that dolphins were more socially active during the day and foraging during the night. Sperm whales were mostly foraging during the day, but they may have been moving to other areas during the night. The most intense shipping noise came from a ferry that passed the platform twice a day. Although beaked whales were expected to be present in the area, so far their biosonar signal has not been conclusively found.
\end{abstract}

\section{Introduction}

The principal aim of the ANTARES collaboration is the operation of a Cherenkov Neutrino telescope [1]. In addition to the optical sensors required for this purpose, an array of 36 hydrophones was installed with the aim of investigating acoustic neutrino detection techniques. This acoustic data stream has been active since 2008 and is managed under AMADEUS [2]. Coincidentally, ANTARES is installed in the Pelagos marine sanctuary, home to many marine mammals species, both whales and dolphins, all of which are acoustically active. The fact that ANTARES is cabled (providing real-time access to data) makes it a unique observation platform for the local marine fauna. The Laboratory of Applied Bioacoustics (LAB) has been analysing the acoustic data, relayed through AMADEUS, since 2010 with a focus especially on sperm whales and dolphins. The ability to study trends in animal presence and a possible relationship with anthropogenic activities for such a long and continuous time frame is unique and is impossible as a purely biological initiative; the costs for installing such a platform just to measure sound levels and to monitor the environment are too high. Opening up platforms such as ANTARES, and for example the NEPTUNE Ocean Observatory (part of Ocean Networks Canada [3]) which offers open access to data from both acoustic and environmental sensors, to marine researchers will not only help to improve marine research but may also help to obtain funding by widening the scope of applications.

Presented here are some of the signal processing techniques that have been used for the acoustic event detection and classification algorithms on data recorded during 2014 at ANTARES. A few examples of both biological and anthropogenic sonar are shown, and daily cetacean activity is evaluated.

^e-mail: mike.vanderschaar@upc.edu 
In the evaluation of cetacean presence or the measurement of shipping noise it has to be taken into account that the hydrophones are installed deep under water. The sound speed profile of the Mediterranean Sea does not have a common sound channel at depth and is mostly refracting upwards [4]. This means that most surface sources, such as dolphins and ships, may have to be on top of the array for the sound to be able to reach the hydrophones. Deep diving animals, such as beaked whales [5] and especially sperm whales [6] who can regularly dive below $1 \mathrm{~km}$ depth, will have a better horizontal detection range particularly when they vocalize downwards.

\section{Bioacoustics at ANTARES}

As part of the ESONET project [7] a server was installed at the ANTARES data centre to analyse the incoming data. For detection a single channel was used, depending on which one was available. The data was run through software developed by the LAB for bioacoustics data analysis called Listening to the Deep Ocean Environment (LIDO [8]). LIDO takes acoustic data in segments (normally aournd 16 seconds in length) and processes these segments as a whole. It includes modules for sound level measurements, acoustic event detection and classification, localization, spectrogram creation, acoustic data compression and other functions.

The LIDO software uses two broad acoustic categories. First are impulsive signals which are broadband in frequency and well defined in time [9]. Examples of impulsive sounds are biosonar, noise from shipping, echo sounders, etc. The second type of signals are named short tonal [10]. These signals are well defined in frequency and can have a duration from half a second to half a minute or longer. They may be frequency modulated. A signal that remains constant over the duration of an entire segment (e.g. power line interference) is removed by the detector (hence "short tonal"). Examples of these events are dolphin whistles, whale calls, fish, and e.g. some ships produce tonal sounds. Many baleen whales produce low frequency sweeps that depending on the time-frequency resolution used for the analysis can be detected by both impulsive and tonal detectors. However, at ANTARES baleen whales cannot be detected due to the hardware configuration where low frequency sound is filtered.

Normally, multiple versions of these detectors are used to operate in specific frequency bands. In some cases, the detector can be specific enough to primarily pick up events from a particular source. When a detector is not sufficient to identify a source, classifiers are used. The main classifier used in LIDO is a Gaussian Mixture Model (GMM [11]). A separate GMM is fit on the feature space of each competing class, with features composed from the time-frequency domains. When a new acoustic event is detected it is passed through each GMM model, and the model with the highest likelihood decides the class. Under this approach a single event can have a high likelihood for multiple models. In that case the software might not take an immediate decision on the class, indicating that one of the high scoring classes may be present. Over time it is likely that the real class will maintain high scores while the other false classes will score lower. Alternatively, all models may have a low score on a new event. When this happens frequently in a short time period these events are flagged and recorded for manual analysis. They can be used to retrain a class or to introduce a new class. At ANTARES classifiers are used for impulsive signals to distinguish between sperm whale and dolphin biosonar, and shipping impulses.

After classification spectrograms and compressed audio streams are created for real-time playback in the software interface. Especially the spectrograms are useful for post-analysis and obtaining 


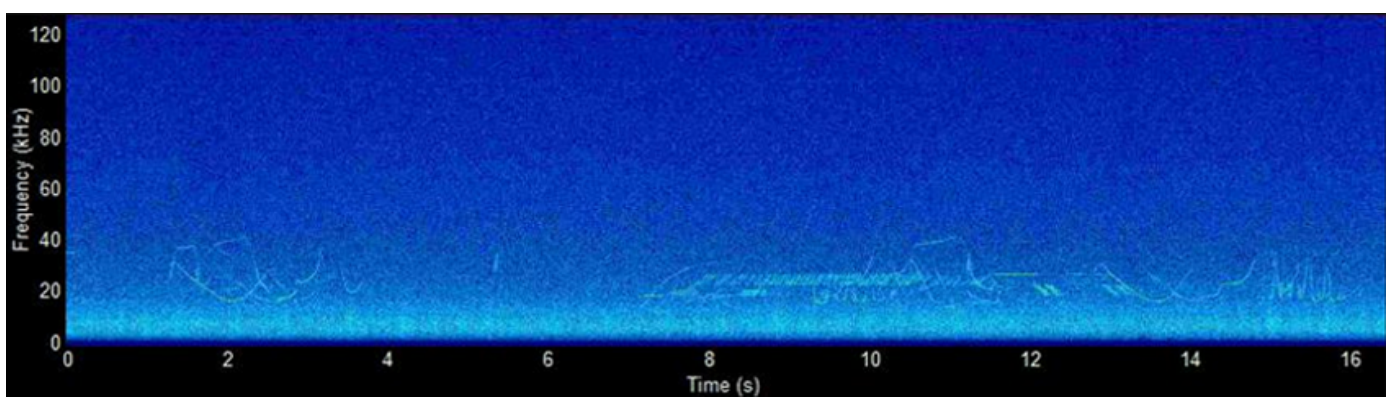

(a)

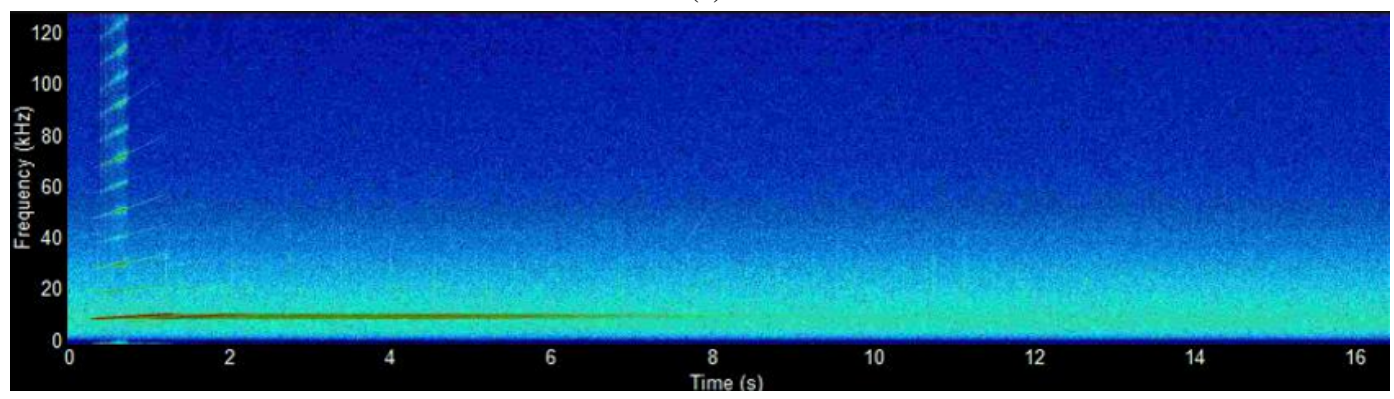

(b)

Figure 1: The top image (a) shows typical dolphin whistles and between 8 and 12 seconds a modulated sound slightly above $20 \mathrm{kHz}$ that is not from a dolphin. The bottom image (b) shows a sonar signal.

a quick overview of the quality of the automatic detection or classification. Sometime multiple spectrograms are created to focus on low and high frequencies separately.

\subsection{Short tonal events}

Short tonal events detected between $2.5-20 \mathrm{kHz}$ at ANTARES were almost always produced by dolphins. Occasionally other signals were encountered such as shown in Fig. (1). On the top image Fig. (1a) there are common dolphin sounds mixed with a frequency modulated tone that continues for a few seconds; the tone might be related to an acoustic release. On the bottom image Fig. (1b) there is signal from what is believed to be a sonar exercise. Fig. (2) shows the short tonal detections throughout the year and for each hour of the day. All histogram frequency axes in this report are provided as percentages representing the percentage of the time that the activity was measured. The monthly histogram Fig. 2a indicates that dolphins were present all year round, with increased activity at the end of the summer and in fall. The hourly image Fig. (2b) suggests that the dolphins were mostly socially active during daylight hours. These findings are currently being studied in more detail.

\subsection{Ultra sonic cetacean clicks}

Ultra sonic cetacean clicks (UCC) are defined as impulses detected between 20 - $46 \mathrm{kHz}$ and classified as UCC. Signals at these frequencies are produced mostly by dolphins and beaked whales. Sperm whale sonar and occasionally impulsive shipping sound is detected in this range as well. For this 


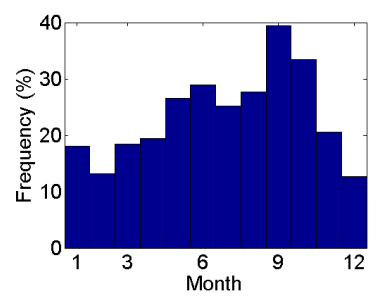

(a)

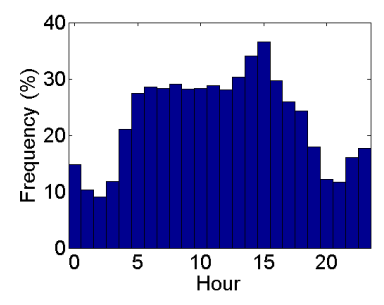

(b)

Figure 2: The histogram on the left (a) shows short tonal activity for each month during 2014 with peak detections at the end of the summer. The histogram on the right (b) shows the short tonal activity for each hour of the day with a higher intensity during daylight hours

reason separate classifiers for these classes were defined. Fig. (3a) shows an example of dolphin biosonar, with impulses around $60 \mathrm{kHz}$ while there is another anthropogenic sonar in the image just below $20 \mathrm{kHz}$. The histogram Fig. 4a) shows the output of the dolphin classifier for each hour during the day. In comparison with the short tonals in Fig. 2b] it is interesting that they peak at different hours, which can be related to the function of the two different signals: biosonar for feeding and whistles for social behaviour.

\subsection{Sperm whale clicks}

Sperm whale presence was evaluated using a classifier as their biosonar signal often overlaps in frequency with shipping generated impulsive noise. An example of a click train from a single animal is shown in the spectrogram in Fig. (3b). The impulses have energy up to $40 \mathrm{kHz}$, but this changes depending on the orientation between the animal and the hydrophone. Surface recordings often record off-axis clicks which have less energy at high frequencies [12]. The histogram Fig. (4b] shows the hourly output of the classifier during 2014 indicating more activity during the day. It may be that the whales forage elsewhere during the night. The presence of sperm whales at ANTARES is studied in more detail in a forthcoming paper.

\subsection{Shipping activity}

The contribution of shipping noise at ANTARES was not found to be very high, except that twice a day a ferry to north Africa would pass by completely saturating the hydrophone acquisition. Due to overlap of other impulsive signals in the shipping band a shipping impulse classifier was trained. Fig. (3c) shows an example of shipping noise. It is clear that low frequency biological sounds are quickly lost in the broadband shipping noise. Higher frequency dolphin biosonar could still be detected in this example. The histogram Fig. (4c) shows the output of the shipping classifier with two peaks in the morning and evening when the ferry passes the platform. Otherwise, most shipping occurred during the day, but for a small percentage of the time. 


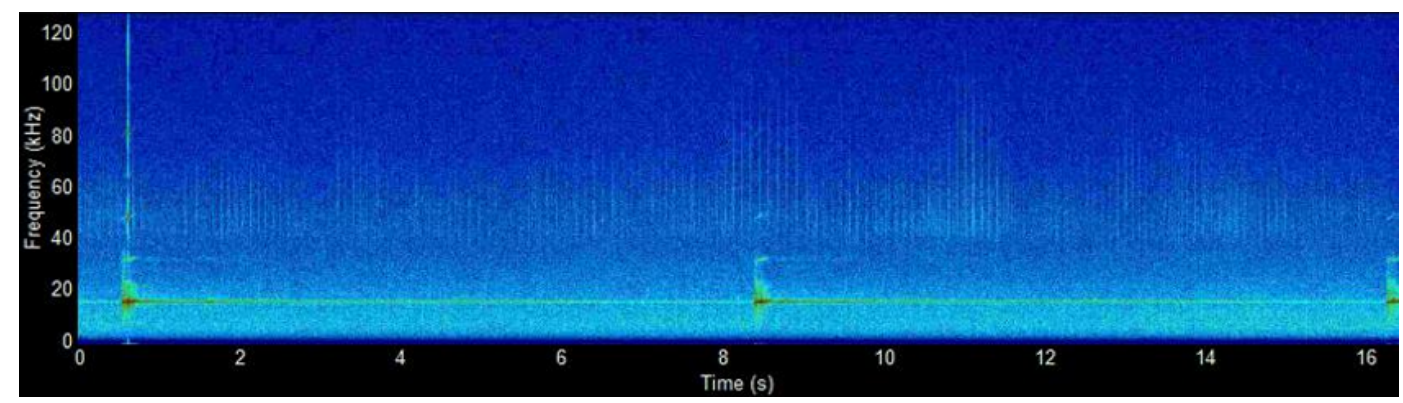

(a)

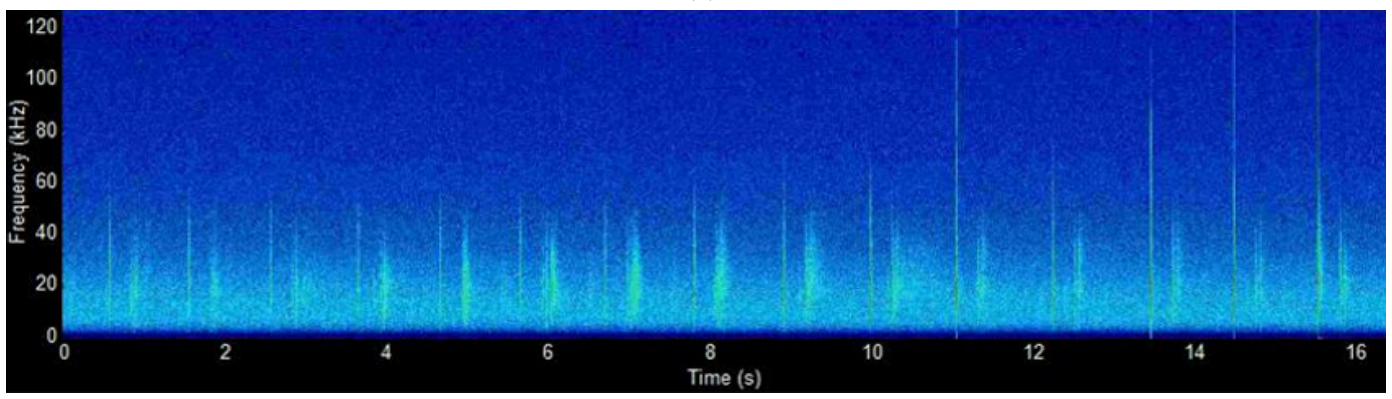

(b)

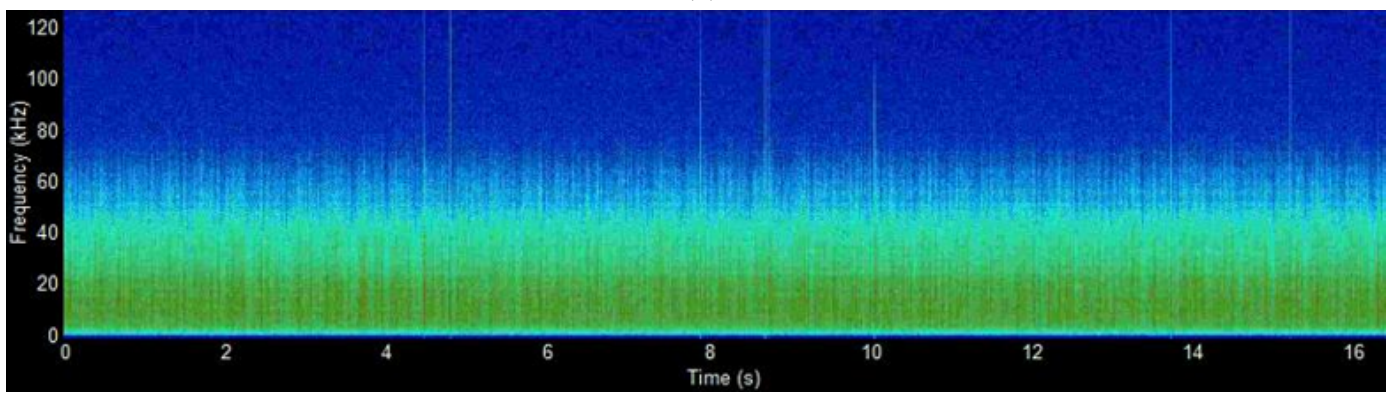

(c)

Figure 3: The top spectrogram (a) is an example of typical high frequency dolphin sonar, visible as the rapid impulses around $60 \mathrm{kHz}$. There is an unknown sonar signal visible around $20 \mathrm{kHz}$. The middle spectrogram (b) shows an example of sperm whale biosonar clicks, with slow pulses up to 40 $\mathrm{kHz}$. The bottom spectrogram (c) shows an example of a ship passing nearby, with both very strong broadband noise and impulsive noise. 


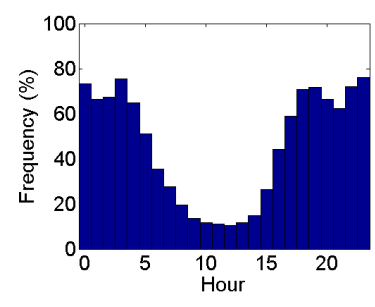

(a)

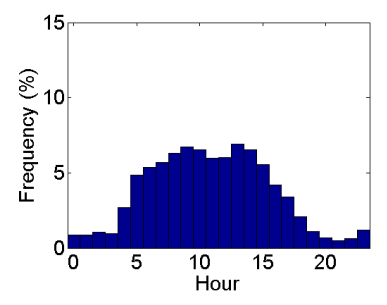

(b)

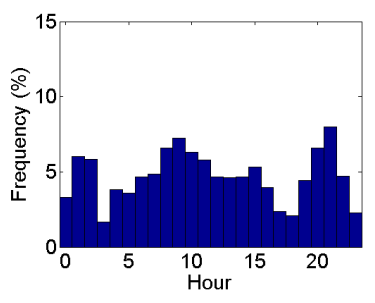

(c)

Figure 4: All histograms show classifier output for each hour of the day during 2014. From left to right the classifiers are: high frequency biosonar (a), sperm whale (b), and shipping (c). Biosonar was especially detected during night, while sperm whales appear to be predominately active during the day. Shipping was mostly present during the day, with a ferry passing by each evening and early morning.

\section{Discussion}

While this paper presented an overview of data analysis of only one year, the strength of platforms such as ANTARES is that data is available during the entire year for many years. This allows establishing baseline levels of cetacean and anthropogenic activities and estimating the common background noise level. Over time, these baseline levels can be tracked and deviations noted. There is especially interest in the increase of background noise levels due to shipping and establishing such a relationship requires many years of data. Even though the position of the hydrophones at ANTARES is not ideal for measuring surface based sources, these deep water platforms can still provide a large amount of unique data. In that sense, it would be advantageous to take into account design requirements of other fields when deploying new monitoring platforms (e.g. KM3). For example, for acoustics it would be preferred not to use hardware high-pass filters and to store or transfer the raw data. This would allow evaluation of the Marine Strategy Framework Directive [13] acoustic criteria.

The results shown here were based on a combination of detectors and classifiers and are considered to be preliminary. In order to verify the monthly or daily patterns such as shown for dolphins between foraging and social behaviour or for sperm whales in their foraging behaviour, a better evaluation of the reliability of the classifiers has to be made. This to ensure that e.g. masking by shipping traffic or anthropogenic impulses misclassified as biosonar are not the reason for reduced or increased detections respectively during a certain time period. This requires manual analysis of the data and analysis results which is currently being done. A more fine-tuned result is expected to be published in 2017.

\section{Acknowledgement}

The main author (MvdS) wishes to thank the organisers of the ARENA 2016 workshop for the invitation to give this presentation. He also wishes to thank his ANTARES colleagues for the fruitful interdisciplinary collaboration. 


\section{References}

[1] J. Aguilar, ANTARES Collaboration, Nuclear Instruments and Methods in Physics Research A 570, 107 (2007)

[2] J. Aguilar, ANTARES Collaboration, Nuclear Instruments and Methods in Physics Research A 626, 128 (2011)

[3] M. Heesemann, T. Insua, M. Scherwath, S. Juniper, K. Moran, Oceanography 27, 151 (2014)

[4] S. Salon, A. Crise, P. Picco, E. de Marinis, O. Gasparini, Annales Geophysicae 21, 833 (2003)

[5] P. Tyack, M. Johnson, N. Aguilar Soto, A. Sturlese, P. Madsen, The Journal of Experimental Biology 209, 4238 (2006)

[6] S. Watwood, P. Miller, M. Johnson, P. Madsen, P. Tyack, Journal of Animal Ecology 75, 814 (2006)

[7] R. Person, L. Beranzoli, C. Berndt, J. Danobitia, M. Diepenbroecke, P. Favali, M. Gillooly, V. Lykousis, J. Miranda, J. Mienert et al., ESONET: An European Sea Observatory Initiative (OCEANS 2008 - MTS/IEEE Kobe Techno-Ocean, 2008), pp. 1-6

[8] M. André, M. van der Schaar, S. Zaugg, L. Houégnigan, A. Sánchez, J. Castell, Journal of the Marine Biological Association 87, 59 (2007)

[9] S. Zaugg, M. van der Schaar, L. Houégnigan, C. Gervaise, A. Sánchez, M. André, Applied Acoustics 71, 1011 (2010)

[10] S. Zaugg, M. van der Schaar, L. Houégnigan, M. André, Applied Acoustics 73, 281 (2012)

[11] C. Bishop, Neural Networks for Pattern Recognition (Oxford university press, 1995)

[12] B. Møhl, M. Wahlberg, P. Madsen, A. Heerfordt, A. Lund, Journal of the Acoustical Society of America 114, 1143 (2003)

[13] European parliament and the council of the European Union, Official Journal of the European Union L164, 19 (2008) 